\title{
Penile Bowenoid Papulosis
}

National Cancer Institute

\section{Source}

National Cancer Institute. Penile Bowenoid Papulosis. NCI Thesaurus. Code C39963.

A cutaneous, usually multifocal lesion in the penis characterized by the presence of verrucous papules. It is caused by human papillomavirus infection. Morphologically there is bowenoid dysplasia present. Progression to invasive carcinoma is infrequent. 